Applied Mathematical Sciences, Vol. 7, 2013, no. 118, 5863 - 5878

HIKARI Ltd, www.m-hikari.com

http://dx.doi.org/10.12988/ams.2013.38430

\title{
The Limit Rotation Loop of a Hyperbolic Plane
}

\author{
Stefano Pasotti \\ DICATAM - Sez. Matematica \\ Università degli Studi di Brescia \\ Via Valotti, 9, 25133 Brescia, Italy \\ Silvia Pianta \\ Dip. Matematica e Fisica \\ Università Cattolica \\ Via Trieste, 17, 25122 Brescia, Italy
}

Copyright (c) 2013 Stefano Pasotti and Silvia Pianta. This is an open access article distributed under the Creative Commons Attribution License, which permits unrestricted use, distribution, and reproduction in any medium, provided the original work is properly cited.

\begin{abstract}
Here we present both an algebraic and a geometric representation of the limit rotation loop build in [7], we interpret its main properties in these settings and we determine its automorphism group.
\end{abstract}

Mathematics Subject Classification: 51M10, 20N05

Keywords: loops, hyperbolic plane, limit rotations, loop sections

\section{Introduction}

This paper is placed in the stream of investigation aiming at describing the relationships between some algebraic structures, such as loops and regular permutation sets, and geometric structures, as hyperbolic geometry and graphs (see e.g. $[2,8,4,14,13])$.

In particular, in the seminal paper [9], the notion of K-loop is used to provide an algebraic representation of a general hyperbolic geometry over an 
euclidean field $\mathbf{K}$, employing a suitable set of motions, namely the point reflections, and [5] provides a sort of coordinatization of a general absolute plane. More recently in [7] the authors together with H. Karzel introduced a class of left conjugacy closed loops which admit a fibration consisting of commutative subsemigroups. A motivating example of such loops arises naturally from a selected subset of limit rotations acting regularly on the point-set $\mathbf{H}$ of a general hyperbolic plane over a Euclidean field $\mathbf{K}$. For the so derived loop $(\mathbf{H}, \oplus)$ it turns out that the left multiplication group is the proper motion group of the plane, isomorphic to $P S L_{2}(\mathbf{K})$ (cf. [7, (3.8.3)], [1]).

Here, starting from the representation of $P G L_{2}(\mathbf{K})$ as the point-set of $P G(3, \mathbf{K})$ deprived of a ruled quadric $\mathcal{Q}$, we characterize the left multiplications of the limit rotation loop $(\mathbf{H}, \oplus)$ as one of the two sheets $\Lambda^{+}$of the tangent cone to $\mathcal{Q}$ through the point $\mathbf{1}$. Moreover we define an isomorphic loop operation directly on the points of the half-cone $\Lambda^{+}$by considering it as an invariant loop section in the group $P S L_{2}(\mathbf{K})$ (following [10] and [11]). In such a way we are also able to determine the automorphism group of the loop $(\mathbf{H}, \oplus)$.

More in details in section 2 we briefly recall the main definitions concerning loops, regular permutation sets and hyperbolic geometry and we summarize the construction of the limit rotation loop as presented in [7].

Section 3 is devoted to present the algebraic model of the limit rotation loop, thus the group $P S L_{2}(\mathbf{K})$ is embedded into the 3-dimensional projective space $\mathrm{PG}(3, \mathbf{K})$ and the set of limit rotations is identified with a cone in such space. Moreover on the points of this cone an orientation is introduced, which turns out to coincide with that introduced in [7]. Finally the operation of the loop is described in terms of the matrices representing limit rotations.

In section 4 a geometric description of the loop operation is provided via the notion of loop section of a group. Moreover we characterize the fibration in commutative subsemigroups and we present a geometric interpretation of the property of the loop of being left conjugacy closed. Finally we characterize the group of automorphisms of the loop among the automorphisms of $P S L_{2}(\mathbf{K})$.

\section{Setting and known results}

Recall that a loop is a non-empty set $P$ endowed with a binary operation "+" such that, for all $a, b \in P$, both equations $a+x=b$ and $y+a=b$ admit a unique solution, and there exists an element $o \in P$, called neutral element, such that $o+a=a=a+o$.

If $(P,+)$ is a loop, then for every element $a \in P$ let $a^{+}: P \rightarrow P$ be the map defined by $a^{+}(x)=a+x$ for every $x \in P$, then it is well known that the set $P^{+}:=\left\{a^{+} \mid a \in P\right\}$ is a subset of $\operatorname{Sym}(P)$ acting regularly on $P$.

Conversely a regular permutation set is a pair $(P, \Gamma)$ where $P$ is a nonempty set and $\Gamma$ is a set of permutations acting regularly on $P$. If $(P, \Gamma)$ is a 
regular permutation set, then $P$ can be equipped with the structure of loop as follows. Fix an element $o \in P$ and, for all $a \in P$ denote by $\widetilde{o, a}$ the unique map of $\Gamma$ mapping $o$ to $a$; then for all $a, b \in P$ define $a+b:=\widetilde{o, a} \circ \widetilde{o, o}(b)$. This construction is called loop derivation of $(P, \Gamma)$ in the point $o$. Note that, if id $\in \Gamma$, then $\widetilde{o, o}=\mathrm{id}$, thus simply $a+b=\widetilde{o, a}(b)$ and in this situation we shall speak of direct loop derivation. In general starting from a loop it is possible to obtain many different regular permutation sets, and conversely many different regular permutation sets can induce on $P$ the same loop structure (see [12] for more details).

In [7] H. Karzel and the two authors introduced a class of fibered loops arising from a suitable subset of the set of limit rotations of a hyperbolic plane via direct loop derivation in the following way. Let $(\mathbf{H}, \mathcal{L}, \alpha, \equiv)$ be a hyperbolic plane over an euclidean field $\mathbf{K}$ and let $\mathfrak{E}$ be the sets of all ends (see $[3, \S 27]$ ). Recall that each proper motion $\mu$ of the plane can be written as a product $\mu=\widetilde{A} \circ \widetilde{B}$, where if $A \in \mathcal{L}$, we denote by $\widetilde{A}$ the line reflection in $A$; a proper motion $\mu=\widetilde{A} \circ \widetilde{B}$ is a limit rotation if the two lines $A$ and $B$ are hyperbolic parallels. In the following we will denote by $\Lambda$ the set of all non-trivial limit rotations of the plane. It is well known that for any pair of distinct points $(a, b) \in \mathbf{H}^{2}$ there exist two limit rotations mapping $a$ to $b$, thus in [7] an orientation is introduced on $\Lambda$ in order to select a regular subset employing the notion of cyclic order. Recall that if we denote by $\mathfrak{E}^{3}$ the set of triples of distinct elements of $\mathfrak{E}$, a cyclic order on the set of ends of a hyperbolic plane (see also [6]) is a map $\zeta: \mathfrak{E}^{3^{\prime}} \rightarrow\{-1,1\},(\alpha, \beta, \gamma) \mapsto \zeta(\alpha, \beta, \gamma)=:[\alpha, \beta, \gamma]$ such that for all distinct $\alpha, \beta, \gamma, \delta \in \mathfrak{E}$ it holds:

(C1) $[\alpha, \beta, \gamma]=[\beta, \gamma, \alpha]=-[\beta, \alpha, \gamma]$

(C2) $[\alpha, \beta, \gamma]=[\alpha, \gamma, \delta] \quad \Longrightarrow \quad[\alpha, \beta, \delta]=[\alpha, \beta, \gamma]$

Now if we fix a triple of ends $\left(\varepsilon, \varepsilon^{\prime}, \infty\right) \in \mathfrak{E}^{3^{\prime}}$, then in [7] the following cyclic order is considered for all $(\alpha, \beta, \gamma) \in \mathfrak{E}^{3^{\prime}}$ :

$$
[\alpha, \beta, \gamma]:=\left[\varepsilon, \varepsilon^{\prime} \mid \gamma, \infty\right] \cdot\left[\varepsilon, \gamma \mid \varepsilon^{\prime}, \beta\right] \cdot[\varepsilon, \alpha \mid \beta, \gamma] \quad \text { for } \quad \beta \neq \varepsilon \neq \gamma \neq \varepsilon^{\prime}
$$

$\left[\varepsilon, \varepsilon^{\prime}, \alpha\right]=\left[\alpha, \varepsilon, \varepsilon^{\prime}\right]=\left[\varepsilon^{\prime}, \alpha, \varepsilon\right]=-\left[\varepsilon^{\prime}, \varepsilon, \alpha\right]=-\left[\alpha, \varepsilon^{\prime}, \varepsilon\right]=-\left[\varepsilon, \alpha, \varepsilon^{\prime}\right]=\left[\varepsilon, \varepsilon^{\prime} \mid \alpha, \infty\right]$ and $[\varepsilon, \gamma, \alpha]=[\alpha, \varepsilon, \gamma]=[\gamma, \alpha, \varepsilon]=-[\gamma, \varepsilon, \alpha]=-[\alpha, \gamma, \varepsilon]=-[\varepsilon, \alpha, \gamma]$, where we define for all $\alpha, \beta, \gamma, \delta \in \mathfrak{E}$ with $\alpha, \beta \neq \gamma, \delta$ :

$$
[\alpha, \beta \mid \gamma, \delta]:= \begin{cases}-1 & \text { if } \alpha \neq \beta, \gamma \neq \delta, \text { and } \overline{\alpha, \beta} \cap \overline{\gamma, \delta} \neq \emptyset \\ 1 & \text { if } \alpha \neq \beta, \gamma \neq \delta, \text { and } \overline{\alpha, \beta} \cap \overline{\gamma, \delta}=\emptyset \\ 1 & \text { if } \alpha=\beta \text { or } \gamma=\delta\end{cases}
$$

and $\overline{\alpha, \beta}$ denotes the unique line of $\mathcal{L}$ defined by the two distinct ends $\alpha$ and $\beta$ (see $[3,(27.2)]$ ). 
If $L$ is a non-trivial limit rotation, $\lambda$ its fixed end and $\alpha$ any end distinct from $\lambda$, then consider the map $\omega: \Lambda \rightarrow\{-1,1\}$ defined by

$$
\omega(L)=[\lambda, \alpha, L(\alpha)]
$$

and write $\Lambda^{+}:=\{L \in \Lambda \mid \omega(L)=1\}$.

In [7] the following is proved.

Theorem 2.1 ([7, (3.2), (3.3), (3.4)] ). For all $L \in \Lambda$ and $A \in \mathcal{L}$ :

1. the map $\omega$ is independent of the choice of the end $\alpha$;

2. $\omega(\widetilde{A} \circ L \circ \widetilde{A})=-\omega(L)$;

3. $\omega\left(L^{-1}\right)=-\omega(L)$;

4. the pair $\left(\mathbf{H}, \Lambda^{+} \cup\{\mathrm{id}\}\right)$ is a regular permutation set.

Exploiting these results, by direct loop derivation, the set $\mathbf{H}$ can be endowed with a loop operation. In particular note that for all $a \in \mathbf{H}$ the map $a^{+}$is the map $\widetilde{o, a}$, thus $\mathbf{H}^{+}=\Lambda^{+} \cup\{\operatorname{id}\}$.

\section{Algebraic Representations of Limit Rotations}

We present here an algebraic representation of the set of limit rotations arising from geometric insight.

Let $(\mathbf{K},+, \cdot)$ be a Euclidean field and identify the elements of the projective linear group $P G L_{2}(\mathbf{K})$ with the points of the projective space of dimension 3 over the field $\mathbf{K}$ deprived of the hyperbolic quadric $\mathcal{Q}$ of equation $x_{1} x_{4}-x_{2} x_{3}=$ 0 through the natural embedding

$$
\mathbf{K}^{*}\left(\begin{array}{ll}
x_{1} & x_{2} \\
x_{3} & x_{4}
\end{array}\right) \longmapsto \mathbf{K}^{*}\left(x_{1}, x_{2}, x_{3}, x_{4}\right) .
$$

Thus the motions of the hyperbolic plane over $\mathbf{K}$, viewed in the Klein model as projectivities of the conic bordering the model, are in one-to-one correspondence with the points of the linear space $\mathrm{PG}(3, \mathbf{K}) \backslash \mathcal{Q}$. In particular, the subgroup of proper motions (represented by classes of matrices with positive determinant and isomorphic to $\left.P S L_{2}(\mathbf{K})\right)$ is identified with one of the two disjoint parts in which the projective space is divided by the quadric $\mathcal{Q}$, namely the one that contains the identity point $\mathbf{1}:=\mathbf{K}^{*}(1,0,0,1)$. Moreover the points of the Klein model itself can be embedded into $\mathrm{PG}(3, \mathbf{K})$ as the internal points of the conic $\mathcal{C}$ obtained as intersection of $\mathcal{Q}$ with the polar plane of the point 1 , of equation $x_{1}+x_{4}=0$. The points of $\mathcal{C}$ are thus of the form:

$$
\mathcal{C}:=\left\{\mathbf{K}^{*} A \mid A \in \mathfrak{M}_{22}(K), \operatorname{det}(A)=0 \wedge \operatorname{tr}(A)=0\right\}
$$


while the points of the Klein model can be represented as

$$
\mathbf{H}:=\left\{[A]:=\{ \pm A\} \in P S L_{2}(\mathbf{K}) \mid \operatorname{tr}(A)=0\right\}
$$

as an easy computation shows. The subgroups of limit rotations correspond to the tangent lines through 1 to $\mathcal{Q}$, and thus are the lines of the quadric cone projecting the conic $\mathcal{C}$ from the point 1 . Again easy computations show that the cone has equation

$$
\left(x_{1}-x_{4}\right)^{2}+4 x_{2} x_{3}=0
$$

and corresponds to the set

$$
\Lambda:=\left\{[L] \in P S L_{2}(\mathbf{K}) \mid \operatorname{tr}(L)=2\right\} .
$$

Note that each element $[L]$ of the set $\Lambda$ acts on $\mathbf{H}$ as a limit rotation by conjugation in the following way:

$$
[L]:\left\{\begin{array}{l}
\mathbf{H} \longrightarrow \mathbf{H} \\
{[X] \longrightarrow\left[L \cdot X \cdot L^{-1}\right] .}
\end{array}\right.
$$

Proposition 3.1. Let $\mathbf{K}^{*} A \in \mathcal{C}$ and $[L] \in \Lambda$ any non-trivial limit rotation. Then the following hold true:

1. the fixed end of the limit rotation $[L]$ is the point $\mathbf{K}^{*}(L-I) \in \mathcal{C}$;

2. the set $\Lambda\left(\mathbf{K}^{*} A\right)$ of all limit rotations with $\mathbf{K}^{*} A$ as fixed end is the subgroup of $P S L_{2}(\mathbf{K})$ :

$$
\Lambda\left(\mathbf{K}^{*} A\right):=\{[k A+I] \in \Lambda \mid k \in \mathbf{K}\} .
$$

Proof. To prove claim 1 note first of all that for all $[L] \in \Lambda$ the matrix $L-I$ has trace 0 and determinant 0 , thus it represents a point of the conic $\mathcal{C}$. Moreover, letting $[L]$ act also on the points of $\mathcal{C}$, we obtain:

$$
[L]\left(\mathbf{K}^{*}(L-I)\right)=\mathbf{K}^{*}\left(L(L-I) L^{-1}\right)=\mathbf{K}^{*}\left(L-L L^{-1}\right)=\mathbf{K}^{*}(L-I),
$$

thus $\mathbf{K}^{*}(L-I)$ is the fixed end of $[L]$.

It is well known that the set of all limit rotations with a fixed end is a subgroup of $P S L_{2}(\mathbf{K})$ (see e.g. [3, (28.10) and (28.11)]). If $\mathbf{K}^{*} A$ is a point of $\mathcal{C}$ then a non-trivial limit rotation $[L]$ fixes $\mathbf{K}^{*} A$ if and only if $L-I=k A$ for a suitable $k \in \mathbf{K}^{*}$, thus the set of all limit rotations fixing $\mathbf{K}^{*} A$ is represented by

$$
\Lambda\left(\mathbf{K}^{*} A\right):=\{[(k A+I)] \mid k \in \mathbf{K}\}
$$

proving 2 . 
If $P=\mathbf{K}^{*} A$ with $A=\left(\begin{array}{ll}a_{1} & a_{2} \\ a_{3} & a_{4}\end{array}\right)$ is any point of the conic $\mathcal{C}$, then $\operatorname{det}(A)=$ $\operatorname{tr}(A)=0$, thus either $a_{2}=0$ and $P$ is the point $P_{\infty}:=\mathbf{K}^{*}\left(\begin{array}{ll}0 & 0 \\ 1 & 0\end{array}\right)$ or $a_{2} \neq 0$ and $P$ is the point $P_{a}:=\mathbf{K}^{*}\left(\begin{array}{cc}-a & -1 \\ a^{2} & a\end{array}\right)$. According to the previous proposition in the following we will denote the subgroups of limit rotations (which, from the geometric point of view, are exactly the lines of $P G(3, \mathbf{K})$ lying on the cone $\Lambda$ ) by:

$$
\begin{gathered}
\Lambda_{\infty}:=\Lambda\left(P_{\infty}\right)=\left\{\left[\left(\begin{array}{ll}
1 & 0 \\
k & 1
\end{array}\right)\right] \in \Lambda \mid k \in K\right\} \\
\Lambda_{a}:=\Lambda\left(P_{a}\right)=\left\{\left[\left(\begin{array}{cc}
1-k a & -k \\
k a^{2} & 1+k a
\end{array}\right)\right] \in \Lambda \mid k \in K\right\} .
\end{gathered}
$$

We may denote a general line of the cone $\Lambda$ by

$$
\Lambda_{a, b}:=\left\{\left[L_{k}\right]=\left[\left(\begin{array}{cc}
1-k a b & -k b^{2} \\
k a^{2} & 1+k a b
\end{array}\right)\right] \in \Lambda \mid k \in \mathbf{K}\right\}
$$

so $\Lambda=\Lambda_{\infty} \cup \bigcup_{a \in \mathbf{K}} \Lambda_{a}=\bigcup_{a, b \in \mathbf{K}} \Lambda_{a, b}$.

Remark 3.2. It is straightforward to verify that each line $\Lambda_{a, b}$ of the cone $\Lambda$ is isomorphic to the additive group of the field $\mathbf{K}$, indeed such lines are exactly the parabolic subgroups of $P S L_{2}(\mathbf{K})$.

We aim now at introducing an orientation on the set $\Lambda$. Denote from now on with $L_{\infty}$ the matrix

$$
L_{\infty}:=\left(\begin{array}{cc}
1 & 0 \\
1 & 1
\end{array}\right)
$$

By definition of $\Lambda$ the minimal polynomial of any matrix $L$, with $[L] \in \Lambda$ is $(x-1)^{2}$, and so it is independent of the limit rotation, thus all such matrices are conjugate, and in particular conjugate to $L_{\infty}$. We need the following preliminary result.

Proposition 3.3. If $[L]$ is a non-trivial limit rotation and $M, N \in \mathrm{GL}(2, \mathbf{K})$ are such that $M L M^{-1}=L_{\infty}=N L N^{-1}$, then $(\operatorname{det} M)(\operatorname{det} N)>0$.

Proof. We distinguish two cases, corresponding to the elements of $\Lambda_{\infty}$ and of $\Lambda_{a}$. 
Let $L_{k}=\left(\begin{array}{ll}1 & 0 \\ k & 1\end{array}\right)$ with $k \neq 0$ and let $M=\left(\begin{array}{ll}m_{1} & m_{2} \\ m_{3} & m_{4}\end{array}\right)$ be any invertible matrix such that $M L_{k} M^{-1}=L_{\infty}$. From here we get

$$
\operatorname{det} M=k m_{4}^{2}
$$

proving that the sign of the determinant of the matrix $M$ depends only on the sign of $k$. obtain:

Let now $L_{k}=\left(\begin{array}{cc}1-k a & -k \\ k a^{2} & 1+k a\end{array}\right)$ with $k \neq 0$ and $M$ as above. Then we

$$
\operatorname{det} M=k\left(a m_{4}-m_{3}\right)^{2}
$$

which shows once again that the sign of the determinant of $M$ is independent of the matrix itself but depends only on the matrix $L_{k}$.

We are now in position to introduce the orientation.

Definition 3.4. Let $[L] \in \Lambda \backslash\{\mathrm{id}\}$ and $M \in \mathrm{GL}(2, \mathbf{K})$ be such that $L_{\infty}=$ $M L M^{-1}$. Then we say that $[L]$ is positive if $\operatorname{det} M>0$, negative otherwise.

According to the previous proposition, this definition makes sense.

Remark 3.5. Note that with the notation of the proof of the previous proposition the limit rotation $\left[L_{k}\right]$ is positive if and only if $k>0$.

Proposition 3.6. The orientation introduced using the matrices as above coincides with the orientation introduced using the map $\omega$ of equation (1).

Proof. In order to compare the orientations let us fix, according to [7], a frame

$$
\varepsilon=\mathbf{K}^{*}\left(\begin{array}{ll}
0 & 1 \\
0 & 0
\end{array}\right), \quad \varepsilon^{\prime}=\mathbf{K}^{*}\left(\begin{array}{ll}
0 & 0 \\
1 & 0
\end{array}\right), \quad \infty=\mathbf{K}^{*}\left(\begin{array}{cc}
1 & 1 \\
-1 & -1
\end{array}\right)
$$

consider a limit rotation $[L] \in \Lambda \backslash\{$ id $\}$ with

$$
L=\left(\begin{array}{cc}
1-k a b & -k b^{2} \\
k a^{2} & 1+k a b
\end{array}\right)
$$

and denote by $\lambda$ the end fixed by $[L]$.

We distinguish several cases.

- If $\lambda=\varepsilon$ (i.e. by Prop. $3.1 a=0, b=1$ ) we have

$$
\omega([L])=\left[\varepsilon, \varepsilon^{\prime},[L]\left(\varepsilon^{\prime}\right)\right]=\left[\varepsilon, \varepsilon^{\prime} \mid[L]\left(\varepsilon^{\prime}\right), \infty\right] .
$$


By direct computation one gets

$$
[L]\left(\varepsilon^{\prime}\right)=\mathbf{K}^{*}\left(\begin{array}{cc}
-k & -k^{2} \\
1 & k
\end{array}\right)
$$

thus the projective lines of $P G(3, \mathbf{K})$ joining $\varepsilon, \varepsilon^{\prime}$ and $[L]\left(\varepsilon^{\prime}\right), \infty$ are respectively:

$$
\begin{gathered}
\overline{\varepsilon, \varepsilon^{\prime}}=\left\{\mathbf{K}^{*}\left(\begin{array}{ll}
0 & 1 \\
h_{1} & 0
\end{array}\right) \in P G L(2, \mathbf{K}) \mid h_{1} \in \mathbf{K}\right\} \\
\overline{[L]\left(\varepsilon^{\prime}\right), \infty}=\left\{\mathbf{K}^{*}\left(\begin{array}{cc}
-k+h_{2} & -k^{2}+h_{2} \\
1-h_{2} & k-h_{2}
\end{array}\right) \in P G L(2, \mathbf{K}) \mid h_{2} \in \mathbf{K}\right\}
\end{gathered}
$$

where $h_{1}, h_{2} \in \mathbf{K}$ are non-homogeneous parameters, and we are assuming $[L]\left(\varepsilon^{\prime}\right) \neq \infty$. The intersection point of these two lines is the point

$$
p=\mathbf{K}^{*} P \quad \text { where } \quad P=\left(\begin{array}{cc}
0 & 1 \\
1 / k & 0
\end{array}\right)
$$

and this point belongs to $\mathbf{H}$ if and only if $\operatorname{det}(P)=-1 / k>0$, if and only if $k<0$, thus

$$
\omega([L])=\left\{\begin{array}{lll}
-1 & \Longleftrightarrow k<0 \\
1 & \Longleftrightarrow k>0 .
\end{array}\right.
$$

If $[L]\left(\varepsilon^{\prime}\right)=\infty$, then we have $k=1>0$ and $\omega([L])=\left[\varepsilon, \varepsilon^{\prime}, \infty\right]=1$ by definition.

- if $\lambda=\varepsilon^{\prime}$ (i.e. by Prop. $3.1 a=1, b=0$ ) we have

$$
\omega([L])=\left[\varepsilon^{\prime}, \varepsilon,[L](\varepsilon)\right]=-\left[\varepsilon, \varepsilon^{\prime} \mid[L](\varepsilon), \infty\right] .
$$

Again by direct computation one gets

$$
[L](\varepsilon)=\mathbf{K}^{*}\left(\begin{array}{cc}
-k & 1 \\
-k^{2} & k
\end{array}\right)
$$

thus

$$
\overline{[L](\varepsilon), \infty}=\left\{\mathbf{K}^{*}\left(\begin{array}{cc}
-k+h_{2} & 1+h_{2} \\
-k^{2}-h_{2} & k-h_{2}
\end{array}\right) \in P G L(2, \mathbf{K}) \mid h_{2} \in \mathbf{K}\right\}
$$

where $h_{2} \in \mathbf{K}$ is a non-homogeneous parameter, and we are assuming $[L](\varepsilon) \neq \infty$. The intersection point of this line with the line $\overline{\varepsilon, \varepsilon^{\prime}}$ expressed as in equation (2) is the point

$$
p=\mathbf{K}^{*} P \quad \text { where } \quad P=\left(\begin{array}{cc}
0 & 1 \\
-k & 0
\end{array}\right)
$$


and this point belongs to $\mathbf{H}$ if and only if $\operatorname{det}(P)=k>0$, thus again

$$
\omega([L])=\left\{\begin{array}{lll}
-1 & \Longleftrightarrow k<0 \\
1 & \Longleftrightarrow k>0 .
\end{array}\right.
$$

If $[L](\varepsilon)=\infty$, then we have $k=-1<0$ and $\omega([L])=\left[\varepsilon^{\prime}, \varepsilon, \infty\right]=-1$ by definition.

- Finally if $\lambda \neq \varepsilon, \varepsilon^{\prime}$ (i.e. by Prop. $3.1 a \neq 0, b \neq 0$ ) we have

$$
\begin{aligned}
\omega([L]) & =\left[\lambda, \varepsilon^{\prime},[L]\left(\varepsilon^{\prime}\right)\right]= \\
& =\left[\varepsilon, \varepsilon^{\prime} \mid[L]\left(\varepsilon^{\prime}\right), \infty\right] \cdot\left[\varepsilon,[L]\left(\varepsilon^{\prime}\right) \mid \varepsilon^{\prime}, \varepsilon^{\prime}\right] \cdot\left[\varepsilon, \lambda \mid \varepsilon^{\prime},[L]\left(\varepsilon^{\prime}\right)\right] .
\end{aligned}
$$

Note that by definition $\left[\varepsilon,[L]\left(\varepsilon^{\prime}\right) \mid \varepsilon^{\prime}, \varepsilon^{\prime}\right]=1$, moreover

$$
[L]\left(\varepsilon^{\prime}\right)=\mathbf{K}^{*}\left(\begin{array}{cc}
-b^{2} k(k a b+1) & -k^{2} b^{4} \\
(1+k a b)^{2} & k b^{2}(1+k a b)
\end{array}\right) .
$$

Proceeding as in the previous cases one gets $\overline{\varepsilon, \varepsilon^{\prime}} \cap \overline{[L]\left(\varepsilon^{\prime}\right), \infty}=\left\{\mathbf{K}^{*} P_{1}\right\}$ and $\overline{\varepsilon, \lambda} \cap \overline{\varepsilon^{\prime},[L]\left(\varepsilon^{\prime}\right)}=\left\{\mathbf{K}^{*} P_{2}\right\}$ where

$$
P_{1}=\left(\begin{array}{cc}
0 & 1 \\
\frac{1+k a b}{k b^{2}} & 0
\end{array}\right) \quad P_{2}=\left(\begin{array}{cc}
-a b & \frac{-k a b^{3}}{1+k a b} \\
a^{2} & a b
\end{array}\right)
$$

These points belong to $\mathbf{H}$ if and only if

$$
\operatorname{det}\left(P_{1}\right)=-\frac{1+k a b}{k}>0 \quad \operatorname{det}\left(P_{2}\right)=-\frac{1}{1+k a b}>0,
$$

thus, by merging this information, one gets:

$$
\omega([L])=\left\{\begin{array}{lll}
-1 & \Longleftrightarrow k<0 \\
1 & \Longleftrightarrow k>0
\end{array}\right.
$$

and by remark 3.5 this completes the proof.

According to the previous result we can itentify $\Lambda^{+}:=\left\{\left[L_{k}\right] \in \Lambda \mid k>0\right\}$ with the positive sheet and $\Lambda^{-}:=\left\{\left[L_{k}\right] \in \Lambda \mid k<0\right\}$ with the negative sheet of the cone $\Lambda$ and observe that for each limit rotation $\left[L_{k}\right] \in \Lambda^{+}$the inverse $\left[L_{k}^{-1}\right]=\left[L_{-k}\right] \in \Lambda^{-}$.

Following [7], exploiting the set of limit rotations $\Lambda^{+} \cup\{\operatorname{id}\}$ and once the point $o=\left[\left(\begin{array}{cc}0 & 1 \\ -1 & 0\end{array}\right)\right] \in \mathbf{H}$ is fixed, on $\mathbf{H}$ we can introduce a loop operation " $\oplus$ " by direct loop derivation in the following way. Consider the map

$$
\varphi:\left\{\begin{array}{lll}
\Lambda & \rightarrow & \mathbf{H} \\
{[A]} & \mapsto & {[A](o)=\left[A\left(\begin{array}{cc}
0 & 1 \\
-1 & 0
\end{array}\right) A^{-1}\right]}
\end{array}\right.
$$


denote by $\varphi_{+}$the restriction of $\varphi$ to $\Lambda^{+} \cup\{$ id $\}$ and note that, by the regularity of $\Lambda^{+} \cup\{$ id $\}$, the map $\varphi_{+}$is bijective and moreover, for all $a \in \mathbf{H}$, we have $\widetilde{o, a}=\varphi_{+}^{-1}(a)$. Then, for all $a, b \in \mathbf{H}$, consider $[A],[B] \in \Lambda^{+} \cup\{$ id $\}$ such that $a=\varphi_{+}([A])$ and $b=\varphi_{+}([B])$ and define

$$
a \oplus b:=\widetilde{o, a}(b)=[A](b)=\left[A B\left(\begin{array}{cc}
0 & 1 \\
-1 & 0
\end{array}\right)(A B)^{-1}\right] .
$$

For any $a \in \mathbf{H}$, denote as in [7] by $\ominus a$ the right inverse and by $\sim a$ the left inverse of $a$, hence if $a=\varphi([A])$ with $[A] \in \Lambda^{+}$, we have

$$
o=a \ominus a=[A](\ominus a), \quad \text { thus } \ominus a=\left[A^{-1}\right](o)=\varphi\left(\left[A^{-1}\right]\right) .
$$

Moreover

$$
o=\sim a \oplus a=\varphi_{+}^{-1}(\sim a)(a),
$$

thus, denoting by $[B]=\varphi_{+}^{-1}(\sim a) \in \Lambda^{+}$, we have

$$
a=\left[B^{-1}\right](o)=\varphi\left(\left[B^{-1}\right]\right) .
$$

Since in the hyperbolic plane $\mathbf{H}$ over $\mathbf{K}$, given any point $a \in \mathbf{H}$ there exist precisely two limit rotations mapping $o$ to $a$, the map $\varphi$ is $2: 1$, hence

$$
\varphi^{-1}(a)=\left\{[A],\left[B^{-1}\right]\right\}
$$

\section{The limit rotation loop as an invariant sec- tion of the group $P S L_{2}(\mathbf{K})$}

Here we want now to provide a geometric description of the loop operation " $\oplus$ " in the projective space representation. In order to do that we need to recall that if $G$ is a group with a subgroup $\mathcal{D}$, a complete set $L$ of representatives of the left cosets of $\mathcal{D}$ in $G$ with $1 \in L$ will be called a transversal of $G / \mathcal{D}$, namely for a transversal $L$ it holds

$$
\forall g \in G: \quad|g \mathcal{D} \cap L|=1 \text { and } 1 \in L .
$$

Following $[10, \S 2 . \mathrm{B}]$ in a transversal $L$ of a coset space $G / \mathcal{D}$ for all $a, b \in L$ there are unique $a * b \in L$ and $d_{a, b} \in \mathcal{D}$ such that $a b=(a * b) d_{a, b}$, thus the transversal $L$ can be equipped with a left-loop operation $a * b:=(a b) d_{a, b}^{-1}$. Moreover the left-loop $(L, *)$ is in fact a loop if and only if for every $g \in G$ it holds that $L$ is a transversal of $g \mathcal{D} g^{-1}$ (see $[10,(2.7)]$ ) and the binary operation "*" on $L$ can be characterized also in set-theoretic terms in the following way:

$$
\forall a, b \in L: \quad a * b=a b \mathcal{D} \cap L .
$$


Consider now the group $P S L_{2}(\mathbf{K})$ acting on the hyperbolic plane over $\mathbf{K}$ and the abelian subgroup of rotations

$$
\mathcal{D}=\left\{\left[\left(\begin{array}{cc}
a & b \\
-b & a
\end{array}\right)\right] \mid a, b \in \mathbf{K}\right\} .
$$

Lemma 4.1. Consider the group $P G L_{2}(\mathbf{K})$ embedded in the projective space $P G(3, \mathbf{K}) \backslash \mathcal{Q}$ as in Section 3 and the cone $\Lambda$ of limit rotations.

1. A line $R$ of $P G(3, \mathbf{K})$ that intersects $\Lambda$ in two points lying on the same sheet of the cone either is a secant line to the quadric $\mathcal{Q}$ or it is a generatrix of the cone;

2. every line not intersecting the quadric $\mathcal{Q}$ meets the positive sheet $\Lambda^{+}$in exactly one point.

Proof. Linear algebra computation.

Since $\mathcal{D}$ is a line of $P G(3, \mathbf{K})$ not intersecting the quadric $\mathcal{Q}$, the same holds for all its left cosets in $P S L_{2}(\mathbf{K})$, which are lines in the projective space, for the left multiplications by elements of $P S L_{2}(\mathbf{K})$ act on $P G(3, \mathbf{K})$ as projectivities fixing $\mathcal{Q}$. Thus, by the previous lemma, the set $\Lambda^{+} \cup\{\mathrm{id}\}$ is a transversal of $P S L_{2}(\mathbf{K}) / \mathcal{D}$ and hence we can equip it with a left-loop operation "*" in the following way:

$$
\forall[A],[B] \in \Lambda^{+} \cup\{\mathrm{id}\}: \quad[A] *[B]=\left[A B D_{A B}^{-1}\right]
$$

for a suitable $\left[D_{A B}\right] \in \mathcal{D}$. Moreover, since for any $[G] \in P S L_{2}(\mathbf{K})$ the line $[G] \mathcal{D}\left[G^{-1}\right]$ does not intersect the quadric $\mathcal{Q}, \Lambda^{+} \cup\{$ id $\}$ turns out to be a transversal also of $[G] \mathcal{D}\left[G^{-1}\right]$, thus “*” turns out to be a loop operation. Geometrically the operation can be seen as follows:

$$
\forall[A],[B] \in \Lambda^{+} \cup\{\operatorname{id}\}: \quad[A] *[B]:=[A B] \mathcal{D} \cap\left(\Lambda^{+} \cup\{\operatorname{id}\}\right) .
$$

This construction provides the required geometric interpretation of the limit rotation loop, in fact the following holds true:

Proposition 4.2. The limit rotation loop $(\mathbf{H}, \oplus)$ and the loop $\left(\Lambda^{+} \cup\{\mathrm{id}\}, *\right)$ are isomorphic.

Proof. The required isomorphism is provided by the map $\varphi_{+}$, in fact for all $[A],[B] \in \Lambda^{+} \cup\{\mathrm{id}\}$ we have

$$
\begin{aligned}
\varphi_{+}([A] *[B]) & =\varphi_{+}\left(\left[A B D_{A B}^{-1}\right]\right)=\left[A B D_{A B}^{-1}\left(\begin{array}{cc}
0 & 1 \\
-1 & 0
\end{array}\right) D_{A B}(A B)^{-1}\right]= \\
& =\left[A B\left(\begin{array}{cc}
0 & 1 \\
-1 & 0
\end{array}\right)(A B)^{-1}\right]=\varphi_{+}([A]) \oplus \varphi_{+}([B]),
\end{aligned}
$$

since $\left[\left(\begin{array}{cc}0 & 1 \\ -1 & 0\end{array}\right)\right] \in \mathcal{D}$ and $\mathcal{D}$ is an abelian group. 
Note that for any $a \in \mathbf{H}$, from $\varphi^{-1}(a)=\left\{[A],\left[B^{-1}\right]\right\}$ we obtain

$$
\left[A\left(\begin{array}{cc}
0 & 1 \\
-1 & 0
\end{array}\right) A^{-1}\right]=\left[B^{-1}\left(\begin{array}{cc}
0 & 1 \\
-1 & 0
\end{array}\right) B\right]
$$

hence $\left[B^{-1}\right] \in[A] \mathcal{D}$, i.e. $\varphi^{-1}(a)=[A] \mathcal{D} \cap \Lambda$.

In [7] for all $a \in \mathbf{H}$ the following centralizer is considered

$$
[a]_{\oplus}:=\left\{x \in \mathbf{H} \mid x^{\oplus} \circ a^{\oplus}=a^{\oplus} \circ x^{\oplus}\right\}
$$

and a technique is described in section 2.1 to enlarge such centralizer to an abelian group $[[a]]_{\oplus}$. Namely we have

$$
[[a]]_{\oplus}:=[a]_{\oplus} \cup \ominus[a]_{\oplus}
$$

where, for any $x \in \mathbf{H}, \ominus x$ denotes the right inverse of $x$ and $\ominus[a]_{\oplus}=\{\ominus x \mid x \in$ $\left.[a]_{\oplus}\right\}$. Since for all $a \in \mathbf{H}$ we have $a^{\oplus}=\varphi_{+}^{-1}(a) \in \Lambda^{+} \cup\{\operatorname{id}\}$, an element $x \in[a]_{\oplus}$ if and only if the limit rotations $\varphi_{+}^{-1}(a)$ and $\varphi_{+}^{-1}(x)$ commute, and this happens precisely if these limit rotations have the same fixed end, or equivalently if they belong to the same line of the cone $\Lambda$. Moreover the group $[[a]]_{\oplus}$ is isomorphic to the centralizer of $a^{\oplus}$ in the full left multiplications group of $(\mathbf{H}, \oplus)$, which is $P S L_{2}(\mathbf{K})$ (cf. [7, Rmk. 2.11]). Thus each enlarged centralizer $[[a]]_{\oplus}$ of the loop $(\mathbf{H}, \oplus)$ corresponds through $\varphi$ to the enlarged centralizer of the element $\varphi_{+}^{-1}(a)=[A] \in \Lambda^{+} \cup\{\mathrm{id}\}$, i.e. the whole line of the cone $\Lambda$ through $[A]$.

Note that, by $[7,(4.1 .1)]$, the loop $(\mathbf{H}, \oplus)$ is left conjugacy closed, and through the isomorphism $\varphi$ this is equivalent to the fact that $\left(\Lambda^{+} \cup\{\mathrm{id}\}\right.$, $\left.*\right)$ is left conjugacy closed, hence

$$
\forall[L] \in \Lambda^{+} \cup\{\operatorname{id}\}: \quad[L]\left(\Lambda^{+} \cup\{\operatorname{id}\}\right)\left[L^{-1}\right]=\Lambda^{+} \cup\{\operatorname{id}\} .
$$

Since, by $[7,(3.8 .3)]$, the set of positive limit rotations $\Lambda^{+}$generates the group of proper motions $P S L_{2}(\mathbf{K})$, then we have also

$$
\forall[G] \in P S L_{2}(\mathbf{K}): \quad[G]\left(\Lambda^{+} \cup\{\operatorname{id}\}\right)\left[G^{-1}\right]=\Lambda^{+} \cup\{\mathrm{id}\} .
$$

thus $\Lambda^{+} \cup\{\mathrm{id}\}$ is an invariant section of $P S L_{2}(\mathbf{K})$ (in the sense of [11]).

From the geometric point of view, exploiting the correspondence among $P G L_{2}(\mathbf{K})$ and $P G(3, \mathbf{K}) \backslash \mathcal{Q}$, for any $[G] \in P S L_{2}(\mathbf{K})$ the inner automorphism $\psi_{G}: P S L_{2}(\mathbf{K}) \rightarrow P S L_{2}(\mathbf{K}) ;[A] \mapsto\left[G A G^{-1}\right]$ is in particular a collineation of the projective space $P G(3, \mathbf{K})$ fixing the quadric $\mathcal{Q}$ and the point $\mathbf{K}^{*} I$.

Conversely, if we write $\Theta=\left\{\theta \in P G L_{4}(\mathbf{K}) \mid \theta(\mathcal{Q})=\mathcal{Q}\right.$ and $\theta\left(\mathbf{K}^{*} I\right)=$ $\left.\mathbf{K}^{*} I\right\}$ and we denote by $\mathcal{R}_{1}$ and $\mathcal{R}_{2}$ the two reguli of the quadric $\mathcal{Q}$, then for 
each $\theta \in \Theta$ either $\theta\left(\mathcal{R}_{1}\right)=\mathcal{R}_{1}$ or $\theta\left(\mathcal{R}_{1}\right)=\mathcal{R}_{2}$; we will write $\Theta^{+}$for the set of $\theta \in \Theta$ fixing each of the two reguli, and $\Theta^{-}$for the set of $\theta \in \Theta$ interchanging $\mathcal{R}_{1}$ and $\mathcal{R}_{2}$. In [15] it is proven that if we denote by $\Psi$ the set of collineations of $P G L_{4}(\mathbf{K})$ corresponding to the inner automorphisms of $P S L_{2}(\mathbf{K})$ and by $\omega$ the map

$$
\omega: \begin{cases}P G(3, \mathbf{K}) & \rightarrow P G(3, \mathbf{K}) \\ \mathbf{K}^{*} A & \mapsto \mathbf{K}^{*} A^{-1},\end{cases}
$$

then $\Theta^{+}=\Psi$ and $\Theta^{-}=\Psi \circ \omega$. If $S$ is a transversal of the left cosets of $\mathcal{D}$ in $P S L_{2}(\mathbf{K})$ which is invariant under the action of $\Theta^{+}$, then the corresponding loop is a left conjugacy closed loop on which every $\theta \in \Theta^{+}$induces a loop automorphism (by $[10,2.7 .6]$ ), thus we are interested in searching for such subsets of the projective space associated to $P S L_{2}(\mathbf{K})$. Some of the loops obtained in this way will be the target of more investigation in forthcoming papers.

\section{Remark 4.3.}

1. Assume that a transversal $S$ equipped with a loop structure is not invariant under the action of $\Theta^{+}=\Psi$, and $[G] \in P S L_{2}(\mathbf{K})$ is such that $\psi_{G}(S)=S^{\prime} \neq S$. Since $S$ is a loop, by [10, (2.7.4.IV) and (2.7.4.III)], $S^{\prime}$ is a transversal of $P S L_{2}(\mathbf{K}) /[G] \mathcal{D}\left[G^{-1}\right]$. Moreover for all $[A] \in P S L_{2}(\mathbf{K})$ it holds $\psi_{A}\left(S^{\prime}\right)=\psi_{A G}(S)$, thus again by [10, (2.7.4)], $\psi_{A}\left(S^{\prime}\right)$ is a transversal of $P S L_{2}(\mathbf{K}) /[G] \mathcal{D}\left[G^{-1}\right]$ and, by the same theorem, $S^{\prime}$ has the structure of a loop. Note that in this situation the isomorphism $\psi_{G}$ fulfils the hypotheses of theorem (2.7.6) of [10], thus the two loops $S$ and $S^{\prime}$ are isomorphic.

2. If, on the other side, the loop $S$ is not invariant under the action of the map $\omega$, then one can consider the transversal $S^{\prime}=\omega(S)$ and equip it with the structure of a loop. Note that, since $S^{\prime}=S^{-1}$ in general the two loops obtained by loop derivation can have a pretty different structure, and can also be non isotopic (see [12, Rmk 2.7.2] for an example). Note in particular that the limit rotation loop is not invariant under the action of $\Theta^{-}$and since for a limit rotation $[L] \in \Lambda$ with

$$
L=\left(\begin{array}{cc}
1-k a b & -k b^{2} \\
k a^{2} & 1+k a b
\end{array}\right)
$$

it holds

$$
L^{-1}=\left(\begin{array}{cc}
1+k a b & k b^{2} \\
-k a^{2} & 1-k a b
\end{array}\right)
$$

then $\omega\left(\Lambda^{+} \cup\{\operatorname{id}\}\right)=\Lambda^{-} \cup\{$ id $\}$. Observe also that the map defined by left cosets of $\mathcal{D}$, namely $\Lambda^{+} \cup\{$ id $\} \rightarrow \Lambda^{-} \cup\{$ id $\} ;[L] \mapsto[L] \mathcal{D} \cap \Lambda^{-} \cup\{$ id $\}$ is not a loop isomorphism by $[10,(2.8 .7)]$. 
We provide now a characterization of the automorphism group of $(\mathbf{H}, \oplus)$ in terms of collineations of $P G(3, \mathbf{K})$. If $\alpha \in \operatorname{Aut}(\mathbf{H}, \oplus)$, since the group generated by $\Lambda^{+} \cup\{\mathrm{id}\}$ is the whole group $P S L_{2}(\mathbf{K})$ by [7, (3.8.3)], the map $\operatorname{Aut}(\mathbf{H}, \oplus) \rightarrow \operatorname{Aut}\left(P S L_{2}(\mathbf{K})\right) ; \alpha \mapsto \widehat{\alpha}$, where

$$
\widehat{\alpha}:\left\{\begin{array}{ccc}
P S L_{2}(\mathbf{K}) & \rightarrow & P S L_{2}(\mathbf{K}) \\
f & \mapsto \quad \alpha \circ f \circ \alpha^{-1},
\end{array}\right.
$$

is a monomorphism by $[10,(2.5)]$.

Proposition 4.4. The group $\operatorname{Aut}(\mathbf{H}, \oplus)$ is isomorphic to the subgroup

$$
T=\left\{\beta \in \operatorname{Aut}\left(P S L_{2}(\mathbf{K})\right) \mid \beta\left(\mathbf{H}^{\oplus}\right)=\mathbf{H}^{\oplus} \text { and } \beta(\mathcal{D})=\mathcal{D}\right\} .
$$

Proof. Let $\alpha \in \operatorname{Aut}(\mathbf{H}, \oplus)$. For all $a, x \in \mathbf{H}$ we have

$$
\left[\widehat{\alpha}\left(a^{\oplus}\right)\right](x)=\alpha \circ a^{\oplus} \circ \alpha^{-1}(x)=\alpha\left(a \oplus \alpha^{-1}(x)\right)=\alpha(a)^{\oplus}(x)
$$

thus $\widehat{\alpha}\left(\mathbf{H}^{\oplus}\right)=\mathbf{H}^{\oplus}$. Moreover for all $[A] \in P S L_{2}(\mathbf{K})$ a point $x \in \mathbf{H}$ is fixed by $[A]$ if and only if the point $\alpha(x)$ is fixed by $\widehat{\alpha}([A])$, thus

$$
\begin{aligned}
\widehat{\alpha}(\mathcal{D}) & =\widehat{\alpha}\left(\left\{[A] \in P S L_{2}(\mathbf{K}) \mid[A](x)=x \Longleftrightarrow x=o\right\}\right)= \\
& =\left\{[A] \in P S L_{2}(\mathbf{K}) \mid[A](x)=x \Longleftrightarrow x=\alpha(o)\right\}=\mathcal{D}
\end{aligned}
$$

since $\alpha(o)=o$, thus $\operatorname{Aut} \widehat{(\mathbf{H}, \oplus)} \subseteq T$.

Conversely let $\beta \in T$. By $[10,(2.7 .6)] \beta \in \operatorname{Aut}\left(\Lambda^{+} \cup\{\operatorname{id}\}, *\right)$, thus by proposition 4.2 the map $\varphi_{+} \circ \beta \circ \varphi_{+}^{-1}$ is $\operatorname{in} \operatorname{Aut}(\mathbf{H}, \oplus)$ and hence we can consider

$$
\chi:\left\{\begin{array}{rlc}
T & \rightarrow & \operatorname{Aut}(\mathbf{H}, \oplus) \\
\beta & \mapsto \varphi_{+} \circ \beta \circ \varphi_{+}^{-1} .
\end{array}\right.
$$

For all $\alpha \in \operatorname{Aut}(\mathbf{H}, \oplus)$ and $x \in \mathbf{H}$ we have

$$
[\chi \circ \widehat{\alpha}](x)=\varphi_{+} \circ \widehat{\alpha} \circ \varphi_{+}^{-1}(x)=\varphi_{+} \circ \widehat{\alpha}\left(x^{\oplus}\right)=\varphi_{+}\left(\alpha(x)^{\oplus}\right)=\alpha(x),
$$

thus $\chi^{-1}=\widehat{\cdot}$, hence $\beta=\widehat{\chi(\beta)}$ proving that $T \subseteq \operatorname{Aut(\mathbf {H},\oplus )}$.

Theorem 4.5. Let $(\mathbf{H}, \oplus)$ be the limit rotation loop of a general hyperbolic plane over an euclidean field $\mathbf{K}$. Then

$$
\operatorname{Aut}(\mathbf{H}, \oplus) \cong \Psi_{\mathcal{D}} \rtimes \overline{\mathbf{K}}
$$

where $\Psi_{\mathcal{D}}$ is the subgroup of $\Psi$ made up of collineations of $P G(3, \mathbf{K})$ derived from inner automorphism corresponding to the elements of the group $\mathcal{D}$ and $\overline{\mathbf{K}}$ is the group of pure semilinear collineations, namely made up of elements $\bar{\alpha} \in \overline{\mathbf{K}}$ such that

$$
\bar{\alpha}: \begin{cases}P G(3, \mathbf{K}) & \rightarrow P G(3, \mathbf{K}) \\ \mathbf{K}^{*}\left(x_{1}, x_{2}, x_{3}, x_{4}\right) & \mapsto \mathbf{K}^{*}\left(x_{1}^{\alpha}, x_{2}^{\alpha}, x_{3}^{\alpha}, x_{4}^{\alpha}\right)\end{cases}
$$

where $\alpha \in \operatorname{Aut}(\mathbf{K},+, \cdot)$. 
Proof. In the group $P S L_{2}(\mathbf{K})$ consider the fibration made up of the centralizers of each element. It is well known that this fibration is characteristic, and, in our representation of the group $P S L_{2}(\mathbf{K})$ as a subset of the pointset of the projective space $P G(3, \mathbf{K})$, it corresponds precisely to the lines of $P G(3, \mathbf{K})$ through the point 1. By [15, props 2.1 and 2.3] the group $\operatorname{Aut}\left(P S L_{2}(\mathbf{K})\right)$ is precisely the subgroup of collineations of the projective space fixing $\mathbf{1}$ and preserving the quadric $\mathcal{Q}$, moreover by $[15$, Thm 1] it holds

$$
\operatorname{Aut}\left(P S L_{2}(\mathbf{K})\right) \cong \Psi \rtimes \overline{\mathbf{K}} .
$$

Hence, according to the previous proposition, the subgroup $T \leqslant \operatorname{Aut}\left(P S L_{2}(\mathbf{K})\right)$ is isomorphic to a subgroup of collineations of $P G(3, \mathbf{K})$, and to prove the statement it remains only to show that $T \cong \Psi_{\mathcal{D}} \rtimes \overline{\mathbf{K}}$. This result follows noticing that:

1. for all $G \in P S L_{2}(\mathbf{K})$ it holds $\psi_{G}(\mathcal{D})=\mathcal{D}$ if and only if $G \in \mathcal{D}$, thus $\Psi_{\mathcal{D}}=\left\{\psi_{G} \in \Psi \mid \psi_{G}(\mathcal{D})=\mathcal{D}\right.$ and $\left.\psi_{G}\left(\Lambda^{+}\right)=\Lambda^{+}\right\}$

2. since the field $\mathbf{K}$ is euclidean, each $\bar{\alpha} \in \overline{\mathbf{K}}$ preserves the ordering in $\mathbf{K}$, thus by 3.5 it preserves the half cone $\Lambda^{+}$; moreover it is straightforward to see that $\bar{\alpha}(\mathcal{D})=\mathcal{D}$.

\section{References}

[1] R. Hartshorne, Geometry: Euclid and beyond, Undergraduate Texts in Mathematics, Springer-Verlag, New York, 2000.

[2] H. Karzel and S. Pianta, Left loops, bipartite graphs with parallelism and bipartite involution sets, Abh. Math. Sem. Univ. Hamburg 75 (2005), 203-214.

[3] H. Karzel, K. Sörensen, and D. Windelberg, Einführung in die Geometrie, Göttingen, 1973.

[4] H. Karzel and M. Marchi, Relations between the K-loop and the defect of an absolute plane, Results Math. 47 (2005), no. 3-4, 305-326.

[5] H. Karzel and M. Marchi, Vectorspacelike representation of absolute planes, J. Geom. 86 (2006), no. 1-2, 81-97 (2007).

[6] H. Karzel and M. Marchi, Introduction of measures for segments and angles in a general absolute plane, Discrete Math. 308 (2008), 220-230. 
[7] H. Karzel, S. Pasotti, and S. Pianta, A class of fibered loops related to general hyperbolic planes, To appear in Aequationes Math., DOI $10.1007 / \mathrm{s} 00010-012-0164-8$.

[8] H. Karzel, S. Pianta, and E. Zizioli, Polar graphs and corresponding involution sets, loops and Steiner triple systems, Results Math. 49 (2006), no. 1-2, 149-160.

[9] H. Karzel and H. Wefelscheid, A geometric construction of the K-loop of a hyperbolic space, Geom. Dedicata 58 (1995), no. 3, 227-236.

[10] H. Kiechle, Theory of K-loops, Lecture Notes in Mathematics, vol. 1778, Springer-Verlag, Berlin, 2002.

[11] P.T. Nagy and K. Strambach, Loops as invariant sections in groups, and their geometry, Canad. J. Math. 46 (1994), no. 5, 1027-1056.

[12] S. Pasotti and E. Zizioli, Loops, regular permutation sets and colourings of directed graphs, Submitted.

[13] S. Pasotti and E. Zizioli, Slid product of loops: a generalization, Submitted.

[14] S. Pasotti and E. Zizioli, Loops with two-sided inverses constructed by a class of regular permutation sets, J. Geom. 100 (2011), no. 1-2, 129-145.

[15] S. Pianta and E. Zizioli, Collineations of geometric structures derived from quaternion algebras, J. Geom. 37 (1990), no. 1-2, 142-152.

Received: August 1, 2013 